\title{
Competition between hydrogen- and halogen bonds: the effect of solvent volume
}

\author{
Jean Lombard, Tanya le Roex and Delia A. Haynes \\ Department of Chemistry and Polymer Science, Stellenbosch University, P. Bag X1, Matieland, 7602, \\ Stellenbosch, Republic of South Africa. *Email: dhaynes@sun.ac.za
}

\section{Supplementary Information}

\section{Contents}

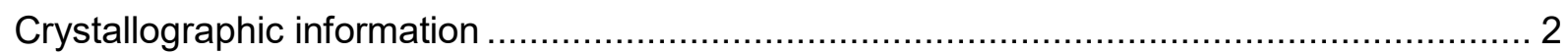

General procedure: Competition experiments ……………….................................... 3

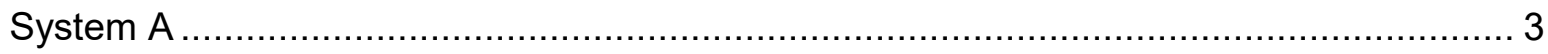

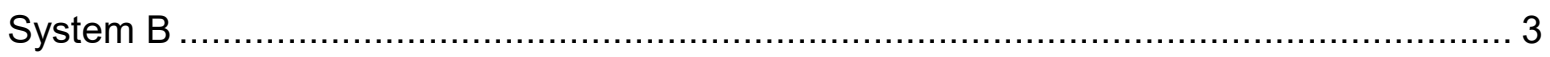

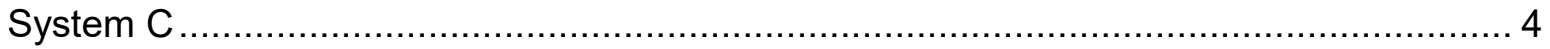

PXRD

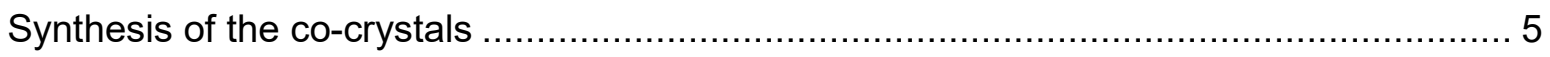

Selectivity Experiments: Mechanochemistry (System A) .............................................. 7

Selectivity Experiments: Mechanochemistry (System B) ............................................... 9

Selectivity Experiments: Mechanochemistry (System C)............................................11

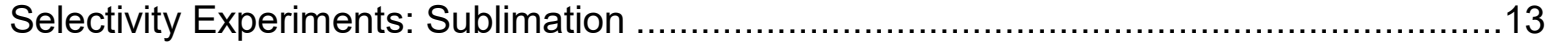

NMR

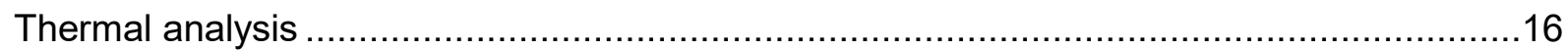




\section{Crystallographic information}

Table S1. Crystallographic information for the co-crystal 1·2a.

\begin{tabular}{|l|c|}
\hline Structure & $\mathbf{1 \cdot 2 a}$ \\
\hline Chemical formula & $\mathrm{C}_{24} \mathrm{H}_{18} \mathrm{~F}_{4} \mathrm{I}_{2} \mathrm{O}_{6}$ \\
Formula weight $/ \mathrm{g} \mathrm{mol}^{-1}$ & 732.18 \\
Crystal system & triclinic \\
Space group & $P \overline{\mathrm{l}}$ \\
Temperature $/ \mathrm{K}$ & $100(2)$ \\
$a / \AA$ & $6.1990(5)$ \\
$b / \AA$ & $8.3411(6)$ \\
$c / \AA$ & $12.5345(9)$ \\
$\alpha /{ }^{\circ}$ & $83.353(1)$ \\
$\beta /{ }^{\circ}$ & $76.046(1)$ \\
$\gamma /{ }^{\circ}$ & $86.037(1)$ \\
Calc. density $/ \mathrm{g} \mathrm{cm}^{-3}$ & 1.948 \\
Volume / $\AA^{3}$ & $624.19(8)$ \\
$Z$ & 1 \\
Independent reflections & 2324 \\
$\mathrm{R}_{\text {int }}$ & 0.0177 \\
$\mathrm{R}_{1}[\mathrm{I}>2 \sigma(\mathrm{I})]$ & 0.0135 \\
\hline
\end{tabular}

Table S2. Hydrogen-bond lengths and angles for $\mathbf{1} \cdot \mathbf{2 a}$ at $100 \mathrm{~K}$.

\begin{tabular}{lllllll}
\hline Structure & $\mathrm{D}-\mathrm{H} \cdots \mathrm{A}$ & $\mathrm{D}-\mathrm{H} / \AA$ & $\mathrm{H} \cdots \mathrm{A} / \AA$ & $\mathrm{D} \cdots \mathrm{A} / \AA$ & $\mathrm{D}-\mathrm{H} \cdots \mathrm{A} /{ }^{\circ}$ & Symmetry code \\
\hline $\mathbf{1 \cdot 2 a}$ & $\mathrm{O} 7-\mathrm{H} 7 \cdots \mathrm{O} 11$ & $0.74(2)$ & $1.99(3)$ & $2.723(2)$ & $171(3)$ & $\mathrm{x}+1, \mathrm{y}, \mathrm{z}$ \\
& $\mathrm{O} 11-\mathrm{H} 11 \cdots \mathrm{O} 15$ & $0.76(3)$ & $1.92(3)$ & $2.667(2)$ & $172(3)$ & \\
& $\mathrm{O} 15-\mathrm{H} 15 \cdots \mathrm{O} 7$ & $0.73(3)$ & $1.96(3)$ & $2.689(1)$ & $171(2)$ & \\
\hline
\end{tabular}

Table S3. Halogen-bond lengths and angles for 1·2a at $100 \mathrm{~K}$.

\begin{tabular}{lllll}
\hline Structure & $\mathrm{Y}-\mathrm{X} \cdots \mathrm{A}$ & $\mathrm{X}-\mathrm{A} / \AA$ & $\mathrm{Y}-\mathrm{X} \cdots \mathrm{A} /{ }^{\circ}$ & Symmetry code \\
\hline $\mathbf{1 \cdot 2 a}$ & $\mathrm{C} 4-\mathrm{I} 1 \cdots \mathrm{O} 11$ & $3.212(1)$ & $157.09(5)$ & $\mathrm{x}, \mathrm{y}+1, \mathrm{z}$ \\
\hline
\end{tabular}




\section{General procedure: Competition experiments}

\section{System A}

The acceptor molecule 1,2-bis(4-pyridyl)ethane (3,21 mg, $0.11 \mathrm{mmol})$ was combined with equimolar quantities of the two donor molecules, 1,4-diiodotetrafluorobenzene (1, $46 \mathrm{mg}, 0.11 \mathrm{mmol})$ and hydroquinone (2a, $13 \mathrm{mg}, 0.11 \mathrm{mmol}$ ). The reactants were combined with $20 \mu 1$ of the relevant solvent $\left(\eta=0.25 \mu \mathrm{mg}^{-1}\right)$ and milled for 20 minutes. The solvents used were toluene, chloroform, dichloromethane, acetone, acetonitrile, 2-propanol, and methanol. For the neat grinding experiments, no solvent was added. The resultant powder was analyzed by powder X-ray diffraction (PXRD).

Slurry experiments were carried out on the same scale as above, but the reactants were added to $0.48 \mathrm{ml}$ of the solvent $\left(\eta=6 \mu \mathrm{mg}^{-1}\right)$. These were added to a small $8 \mathrm{ml}$ glass vial which was capped and sealed with parafilm. The vial was then sonicated in a water bath for a total of 5 minutes (in intervals of 30 seconds, with 30 seconds rest in between to prevent heating of the sample). The solid product was filtered immediately and analyzed by PXRD, the solvent was not simply left to evaporate.

For the sublimation experiments the reagents were added to a thin Schlenk tube $(14 \mathrm{~mm}$ diameter, $220 \mathrm{~mm}$ length) under static vacuum $(\sim 0.6 \mathrm{mbar}$ line pressure $)$, and then heated at $110^{\circ} \mathrm{C}$ or $130^{\circ} \mathrm{C}$ by suspension in an oil bath for 19 hours. Crystals formed on the sides of the glass tube in bands. Each band was removed separately for analysis by PXRD or single-crystal X-ray diffraction (SCXRD).

\section{System $B$}

The acceptor molecule 1,2-bis(4-pyridyl)ethane (3,21 mg, $0.11 \mathrm{mmol})$ was combined with equimolar quantities of the two donor molecules, 1,4-diiodotetrafluorobenzene (1, $45 \mathrm{mg}, 0.11 \mathrm{mmol})$ and 2fluorohydroquinone (2b, $14 \mathrm{mg}, 0.11 \mathrm{mmol}$ ). The reactants were combined with $20 \mu \mathrm{l}$ of the relevant solvent $\left(\eta=0.25 \mu \mathrm{mg}^{-1}\right)$ and milled for 20 minutes. The solvents used were toluene, chloroform, dichloromethane, acetone, acetonitrile, 2-propanol, and methanol. For the neat grinding experiments, no solvent was added. The resultant powder was analyzed by PXRD.

Slurry experiments were carried out on the same scale as above, but the reactants were added to $0.48 \mathrm{ml}$ of the solvent $\left(\eta=6 \mu \mathrm{mg}^{-1}\right)$. These were added to a small $8 \mathrm{ml}$ glass vial which was capped and sealed with parafilm. The vial was then sonicated in a water bath for a total of 5 minutes (in intervals of 30 seconds, with 30 seconds rest in between to prevent heating of the sample). The solid product was filtered immediately and analyzed by PXRD, the solvent was not simply left to evaporate.

For the sublimation experiments the reagents were added to a thin Schlenk tube $(14 \mathrm{~mm}$ diameter, $220 \mathrm{~mm}$ length) under static vacuum ( 0.6 mbar line pressure), and then heated at $110^{\circ} \mathrm{C}$ or $130{ }^{\circ} \mathrm{C}$ by suspension in an oil bath for 19 hours. Crystals formed on the sides of the glass tube in bands. Each band was removed separately for analysis by PXRD or SCXRD. 


\section{System C}

Acceptor molecule 1,2-bis(4-pyridyl)ethane $(3,19 \mathrm{mg}, 0.10 \mathrm{mmol})$ was combined with equimolar quantities of the two donor molecules, 1,4-diiodotetrafluorobenzene (1, $42 \mathrm{mg}, 0.10 \mathrm{mmol})$ and 2,3,5,6tetrafluorohydroquinone $(\mathbf{2 c}, 19 \mathrm{mg}, 0.10 \mathrm{mmol})$. The reactants were combined with $20 \mu \mathrm{l}$ of the relevant solvent $\left(\eta=0.25 \mu \mathrm{mg}^{-1}\right)$ and milled for 20 minutes. The solvents used were toluene, chloroform, dichloromethane, acetone, acetonitrile, 2-propanol, and methanol. For the neat grinding experiments, no solvent was added. The resultant powder was analyzed by PXRD.

Slurry experiments were carried out on the same scale as above, but the reactants were added to $0.48 \mathrm{ml}$ of the solvent $\left(\eta=6 \mu 1 \mathrm{mg}^{-1}\right)$. These were added to a small $8 \mathrm{ml}$ glass vial which was capped and sealed with parafilm. The vial was then sonicated in a water bath for a total of 5 minutes (in intervals of 30 seconds, with 30 seconds rest in between to prevent heating of the sample). The solid product was filtered immediately and analyzed by PXRD, the solvent was not simply left to evaporate.

For the sublimation experiments the reagents were added to a thin Schlenk tube (14 mm diameter, $220 \mathrm{~mm}$ length) under static vacuum ( $\sim 0.6 \mathrm{mbar}$ line pressure $)$, and then heated at $110{ }^{\circ} \mathrm{C}$ or $130{ }^{\circ} \mathrm{C}$ by suspension in an oil bath for 19 hours. Crystals formed on the sides of the glass tube in bands. Each band was removed separately for analysis by PXRD or SCXRD.

Table S4. Properties of solvents used in this study. ${ }^{1}$

\begin{tabular}{|l|l|l|l|l|}
\hline Solvent & $\begin{array}{l}\text { Relative } \\
\text { polarity }\end{array}$ & $\begin{array}{l}\text { Dielectric } \\
\text { constant }\end{array}$ & $\begin{array}{l}\text { Boiling point } \\
\left({ }^{\circ} \mathrm{C}\right)\end{array}$ & $\begin{array}{l}(\text { Purity } \\
\text { grade, \%) }\end{array}$ \\
\hline Toluene & 0.099 & 2.38 & 110.6 & 99.5 \\
\hline Chloroform & 0.259 & 4.89 & 61.2 & 99.9 \\
\hline Dichloromethane & 0.309 & 8.93 & 39.6 & 99.9 \\
\hline Acetone & 0.355 & 20.56 & 56.1 & 99.5 \\
\hline Acetonitrile & 0.460 & 35.94 & 81.6 & 99.8 \\
\hline Isopropanol & 0.546 & 19.92 & 82.2 & 99.7 \\
\hline Methanol & 0.762 & 32.66 & 64.5 & 99.9 \\
\hline
\end{tabular}




\section{PXRD}

\section{Synthesis of the co-crystals}

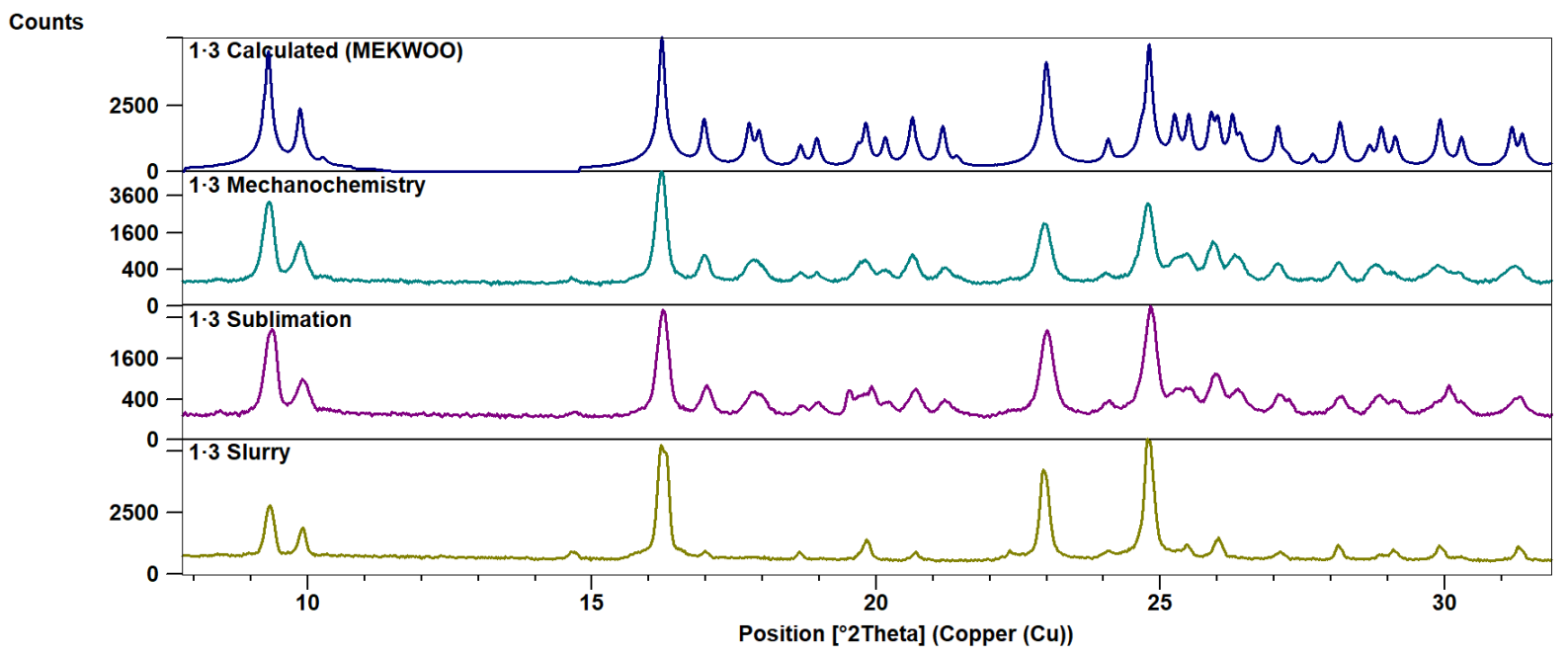

Figure S1. Comparison of the simulated powder pattern for the halogen-bonded co-crystal $1 \cdot 3$ with experimental patterns obtained when $\mathbf{1 \cdot 3}$ is made mechanochemically, by sublimation, and from a slurry. There is good agreement between the patterns, indicative of the formation of pure products.

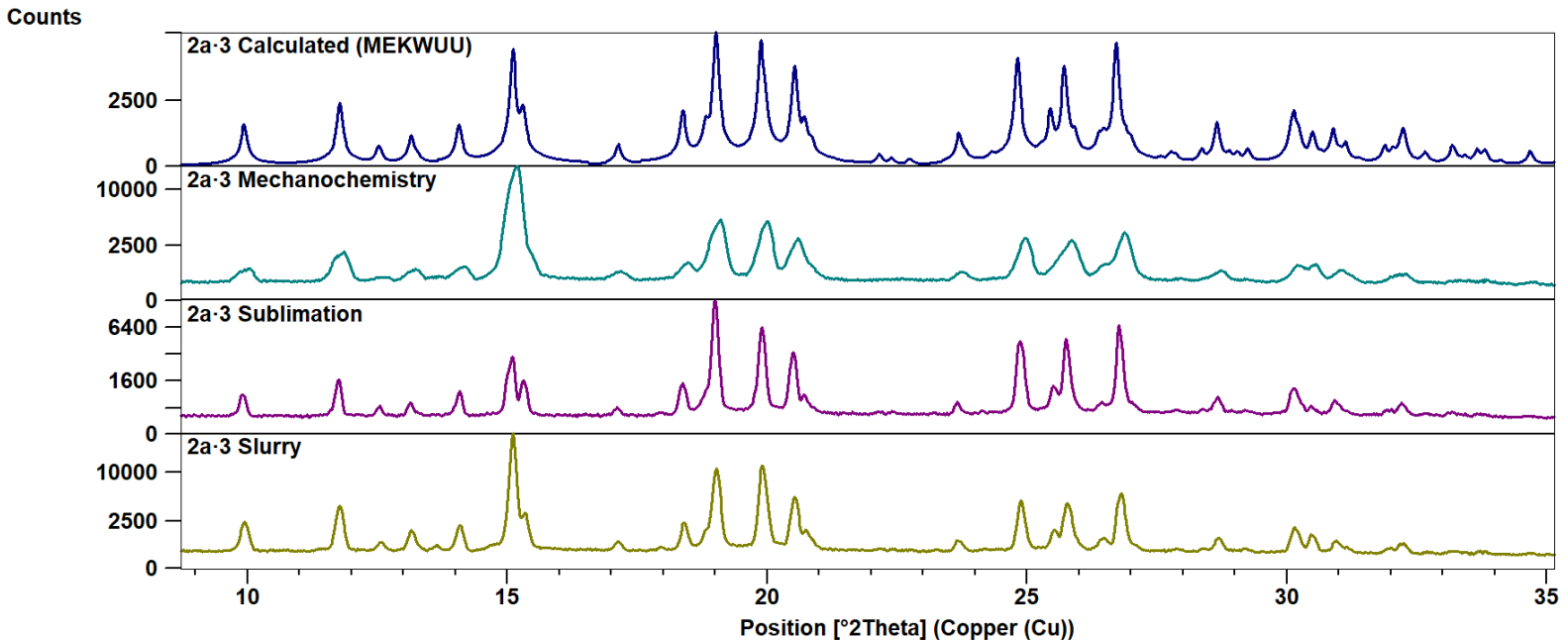

Figure S2. Comparison of the simulated powder pattern for the hydrogen-bonded co-crystal $\mathbf{2 a} \cdot \mathbf{3}$ with experimental patterns obtained when $\mathbf{2 a} \cdot \mathbf{3}$ is made mechanochemically, by sublimation, and from a slurry. There is good agreement between the patterns, indicative of the formation of pure products. 


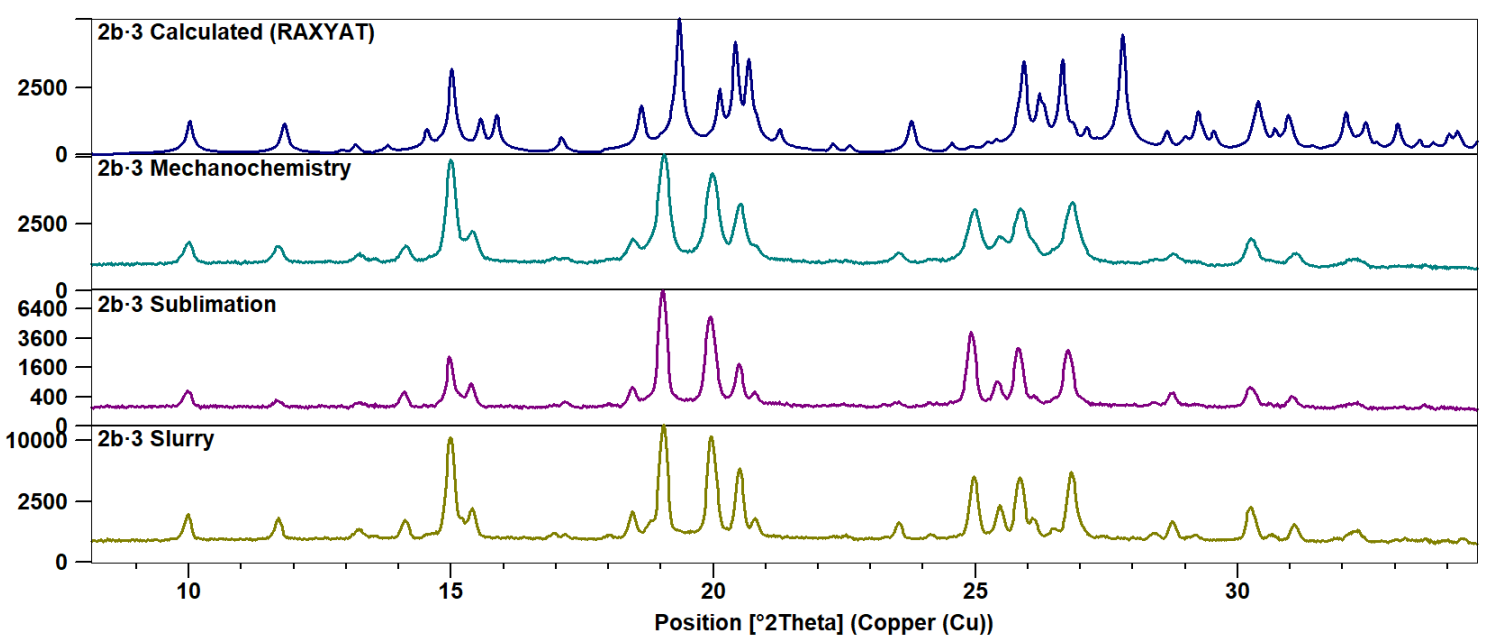

Figure S3. Comparison of the simulated powder pattern for the hydrogen-bonded co-crystal $\mathbf{2} \mathbf{b} \cdot \mathbf{3}$ with experimental patterns obtained when $\mathbf{2 b} \cdot \mathbf{3}$ is made mechanochemically, by sublimation, and from a slurry. There is good agreement between the patterns, indicative of the formation of pure products.

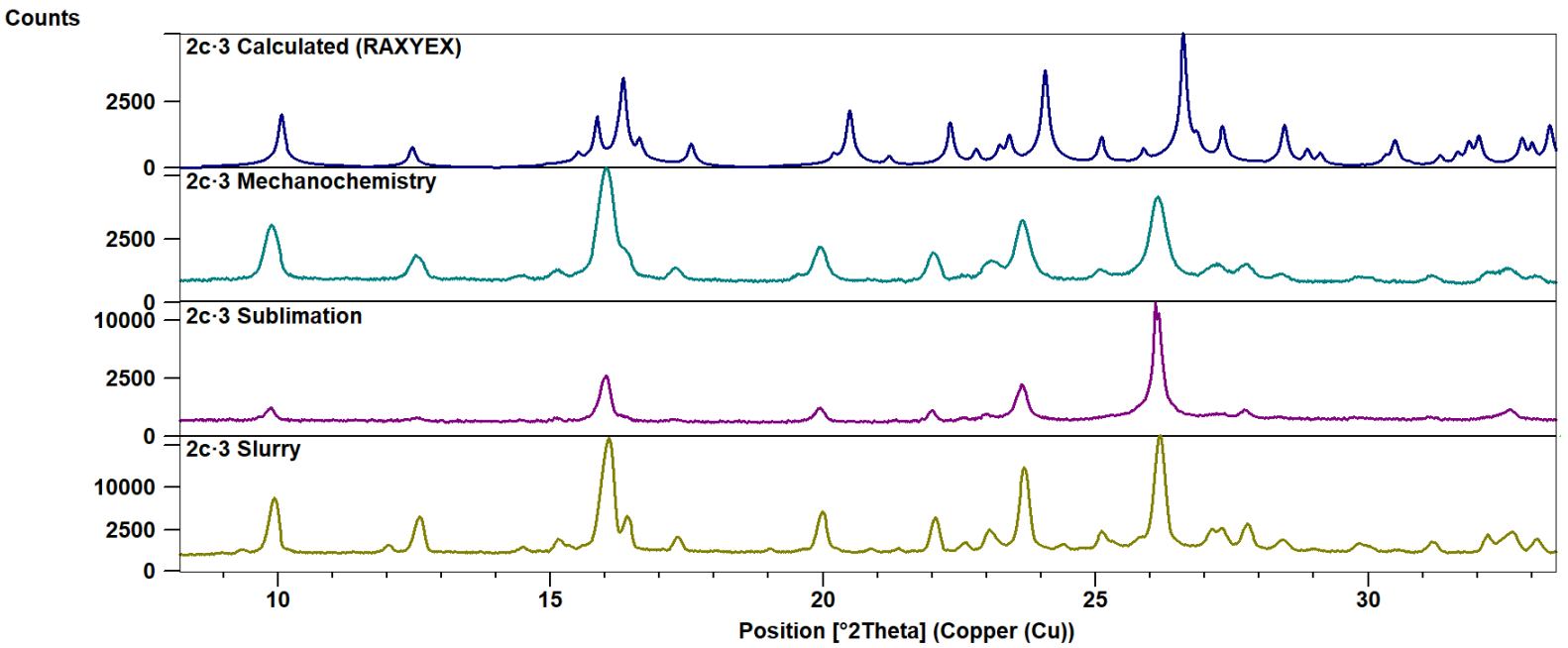

Figure S4. Comparison of the simulated powder pattern for the hydrogen-bonded co-crystal $2 \mathbf{c} \cdot \mathbf{3}$ with experimental patterns obtained when $\mathbf{2 c} \cdot \mathbf{3}$ is made mechanochemically, by sublimation, and from a slurry. There is good agreement between the patterns, indicative of the formation of pure products. 


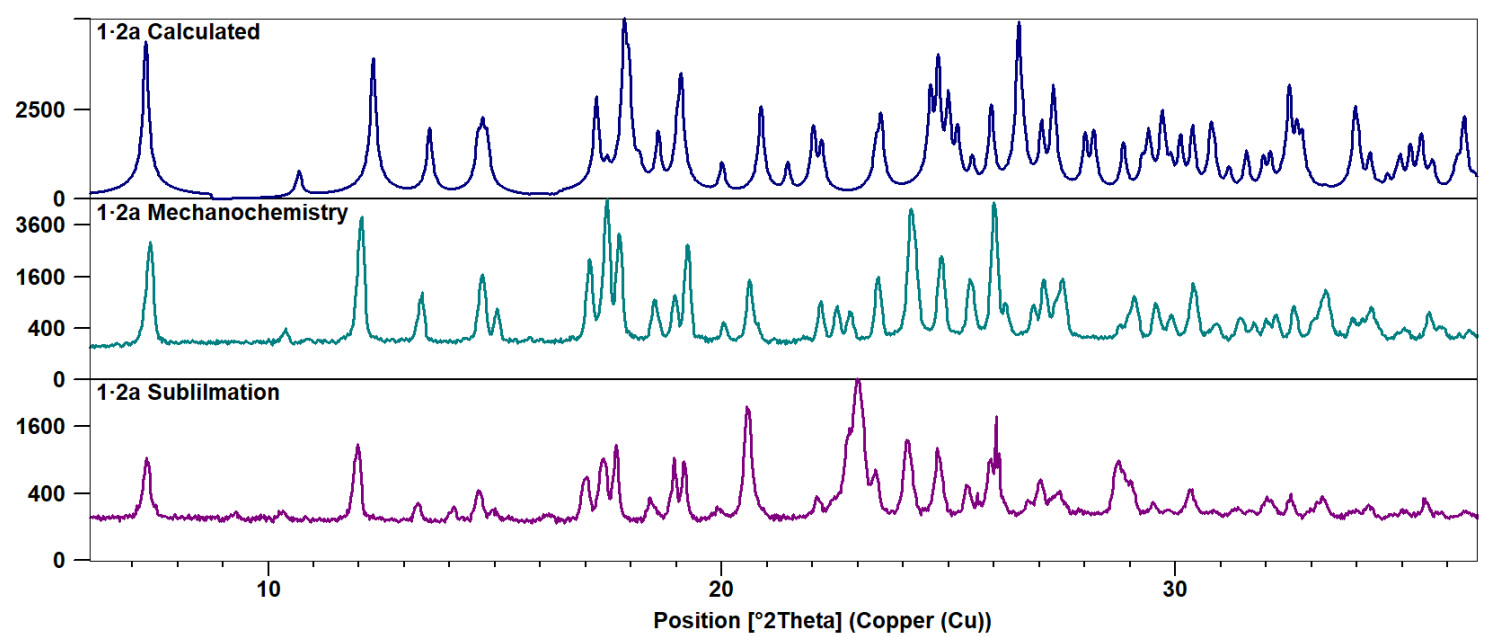

Figure S5. Comparison of the simulated powder pattern for the new co-crystal $(\mathbf{1} \cdot \mathbf{2 a}$ at $100 \mathrm{~K})$ with experimental patterns obtained when $\mathbf{1 \cdot 2 a}$ is made mechanochemically and by sublimation. There is good agreement between the patterns, indicative of the formation of pure products.

\section{Selectivity Experiments: Mechanochemistry (System A)}

Counts

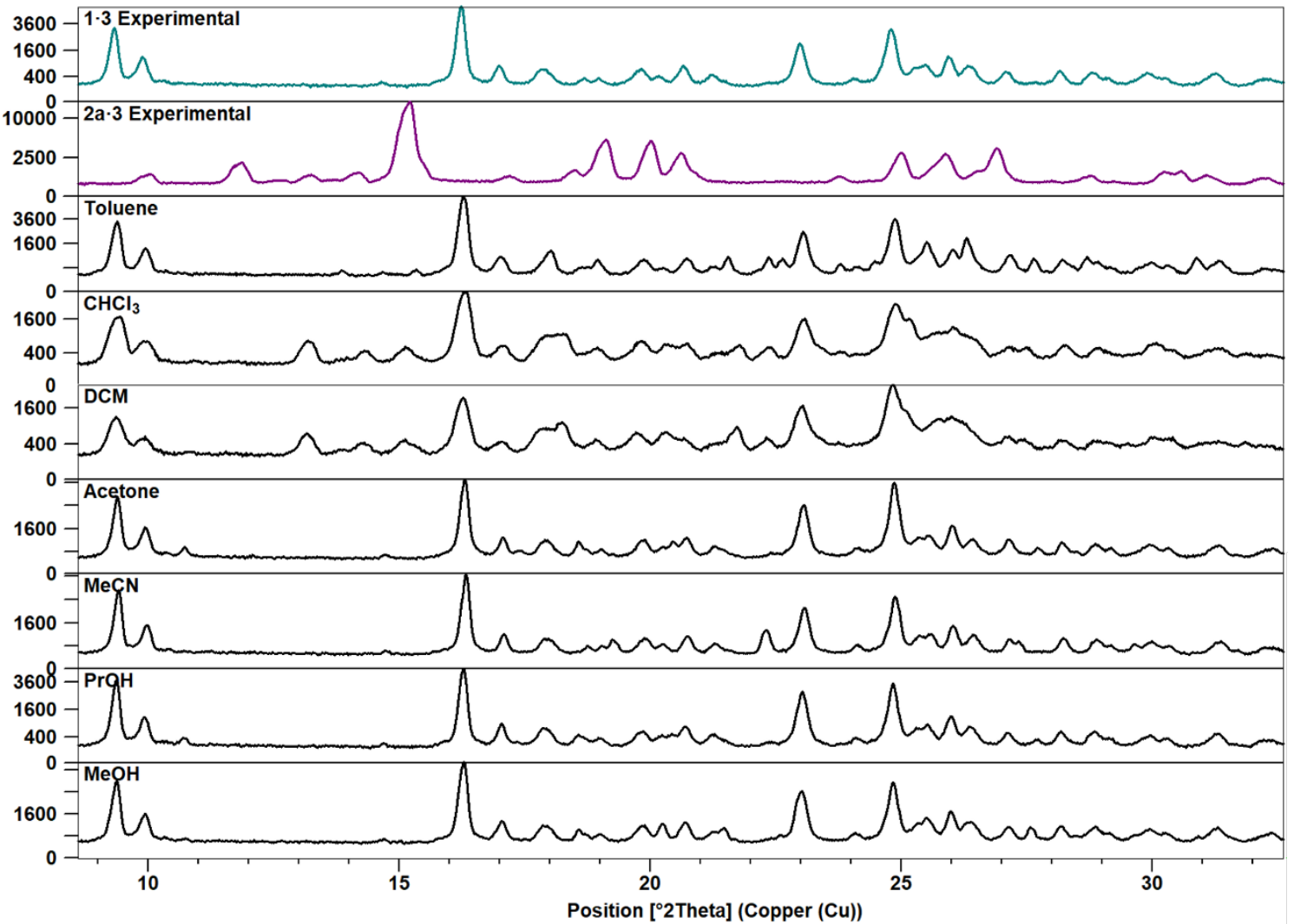

Figure S6. Comparison of LAG results, using seven different solvents, for System A. For each solvent used, the halogen-bonded co-crystal $\mathbf{1 \cdot 3}$ is always formed. When chloroform or dichloromethane is used, additional peaks can be seen. Some of these weak peaks match the hydrogen-bonded co-crystal $2 \mathrm{a} \cdot 3$ (e.g. at $15.2^{\circ}$ ), but a number of peaks do not match either co-crystal, these can be attributed to the ternary co-crystal $\mathbf{1} \cdot \mathbf{2 a} \cdot \mathbf{3}$ (see also Figure S8). 


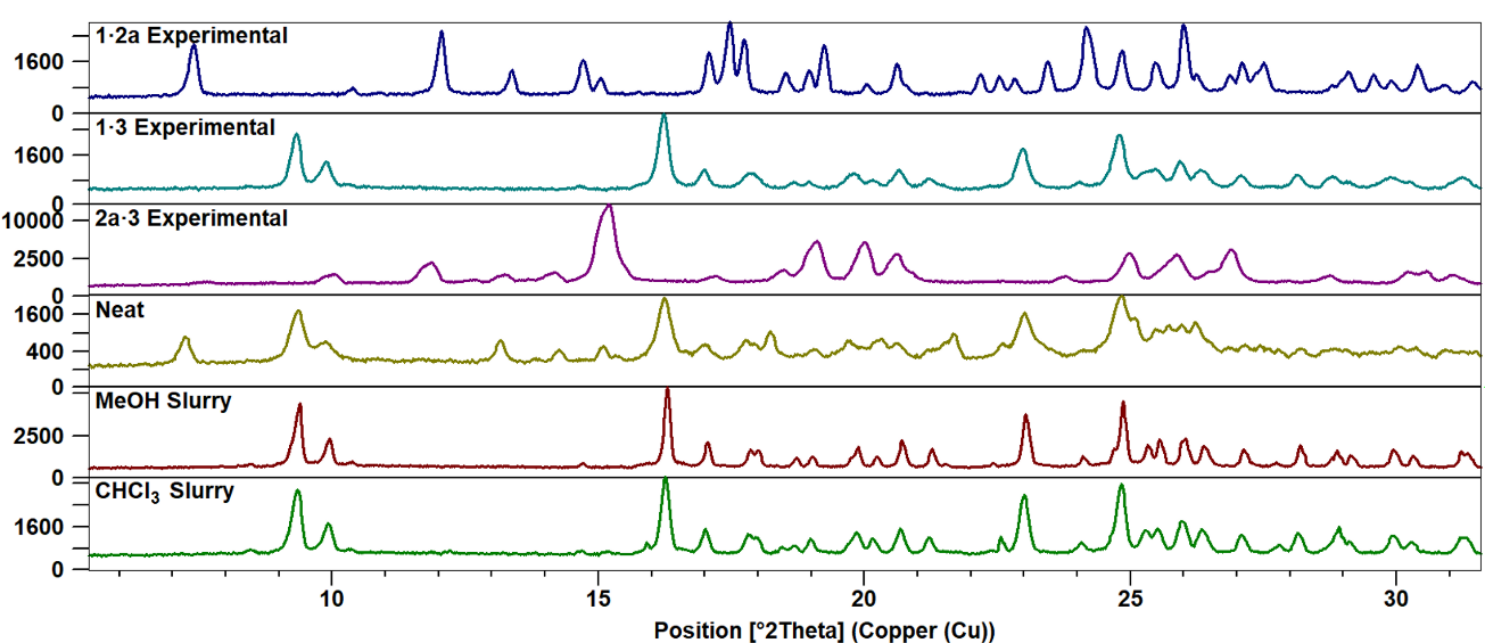

Figure S7. Comparison of neat grinding and slurry results for System A. Similar to the LAG results, the halogen-bonded co-crystal $\mathbf{1} \cdot \mathbf{3}$ is formed each time. When the three starting materials are milled without the addition of any solvent, the same pattern is obtained as with the $\mathrm{DCM}$ and $\mathrm{CHCl}_{3} \mathrm{LAG}$ experiments, i.e. containing $\mathbf{1} \cdot \mathbf{3}, \mathbf{2 a} \cdot \mathbf{3}$, and the ternary co-crystal (see also Figure S8).

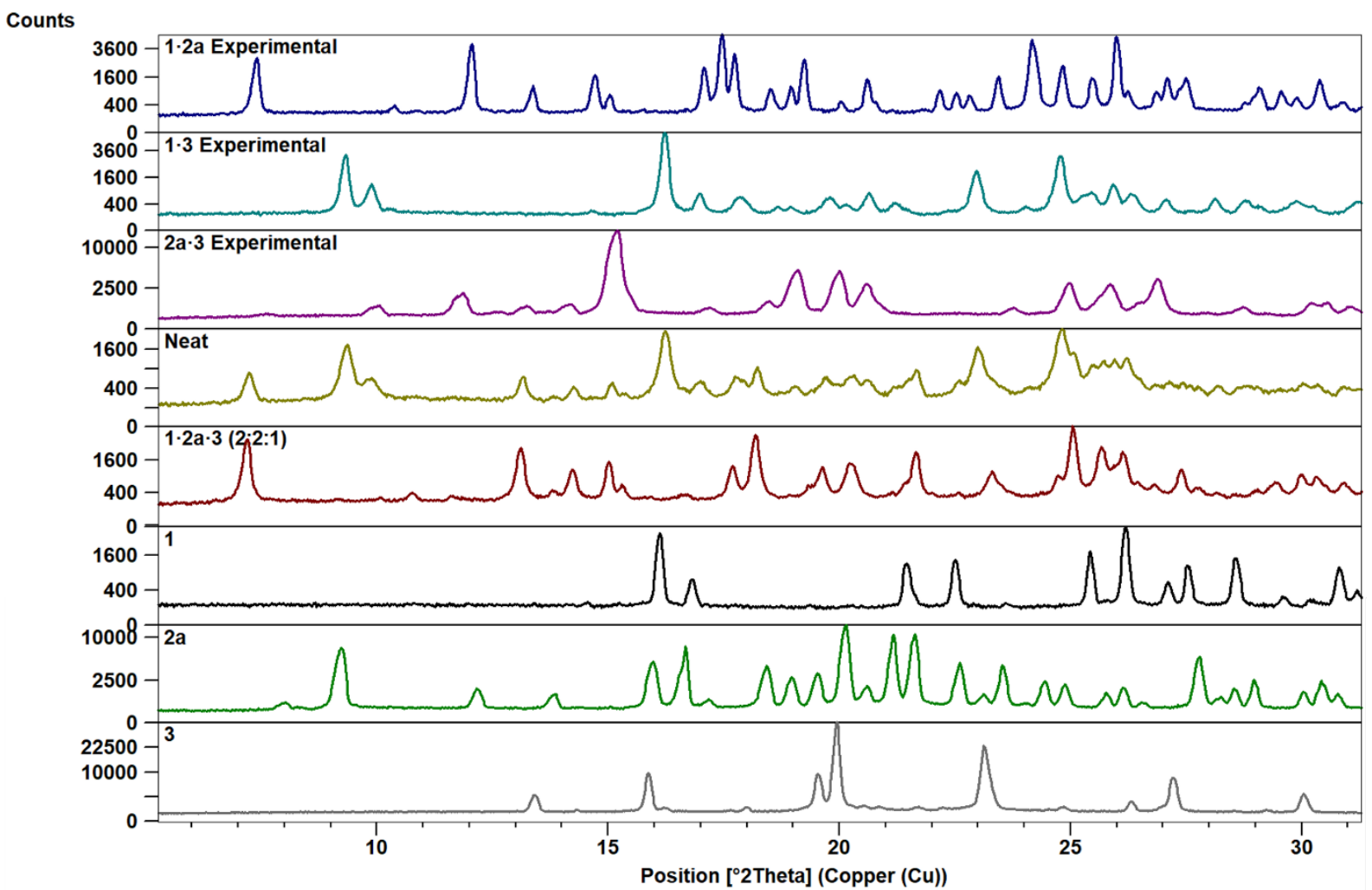

Figure S8. A closer look at one of the patterns containing the ternary co-crystal $1 \cdot 2 a \cdot 3-$ the pattern obtained from neat grinding $\mathbf{1}, \mathbf{2 a}$ and $\mathbf{3}$ is used here as an example, shown in yellow (also contains $\mathbf{1} \cdot \mathbf{3}$ and $\mathbf{2 a} \cdot \mathbf{3}$ ). We suspect that the ternary co-crystal contains $\mathbf{1}, \mathbf{2 a}$, and $\mathbf{3}$ in a 2:2:1 ratio. When the three starting materials are milled in this ratio, the pattern shown in red is obtained, which shows good correlation with peaks in the neat grinding trace that cannot be attributed to any of the binary co-crystals. 


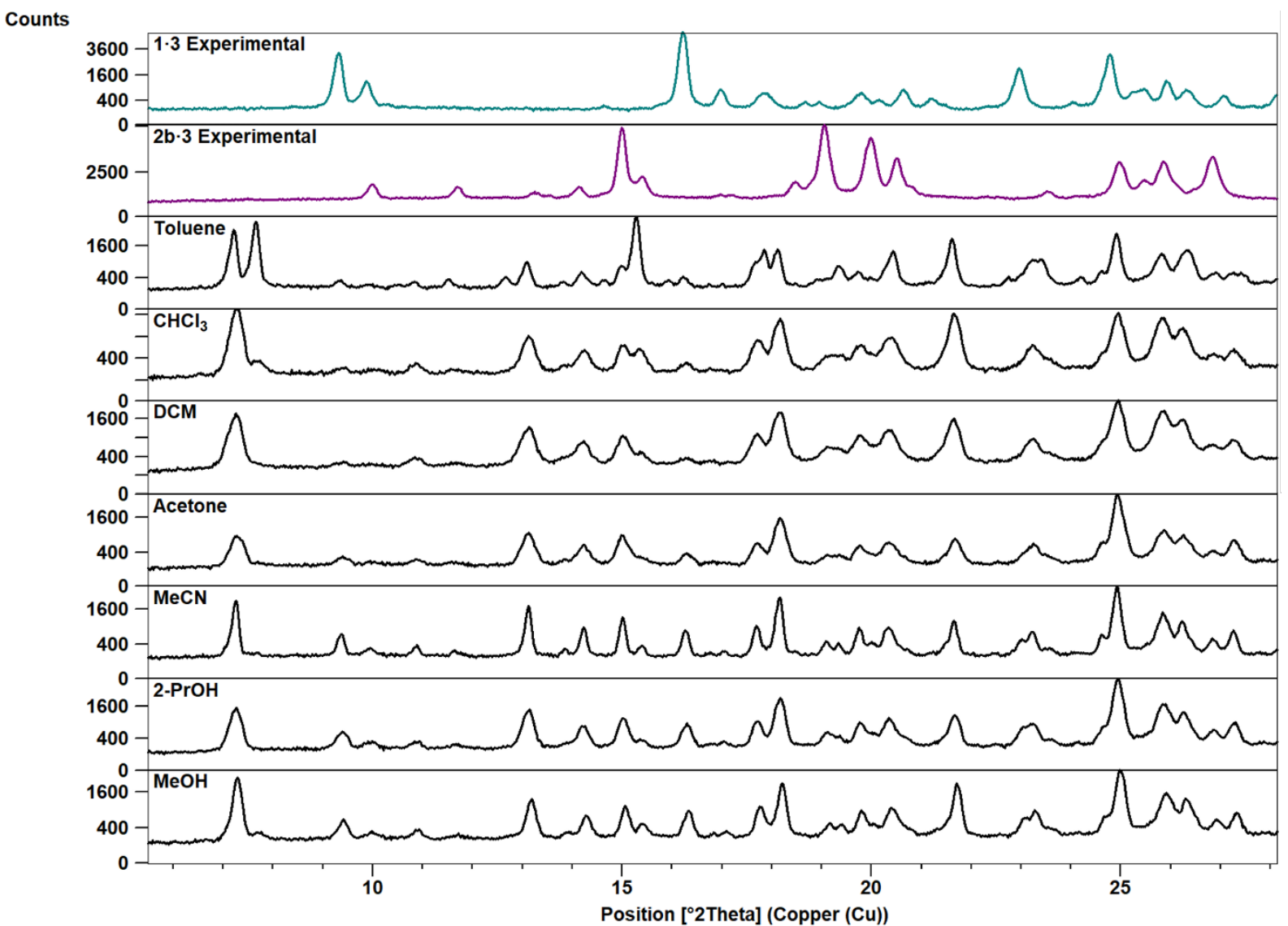

Figure S9. Comparison of LAG results for System B. For each solvent used, a small amount of both the halogen-bonded and hydrogen-bonded co-crystal is always formed, but mostly, peaks of an unknown product can be seen, which has been identified as a ternary co-crystal, $\mathbf{1} \cdot \mathbf{2 b} \cdot \mathbf{3}$ (see also Figure S11). As expected, the more polar the solvent is, the more distinct the $\mathbf{1 \cdot 3}$ peaks are.

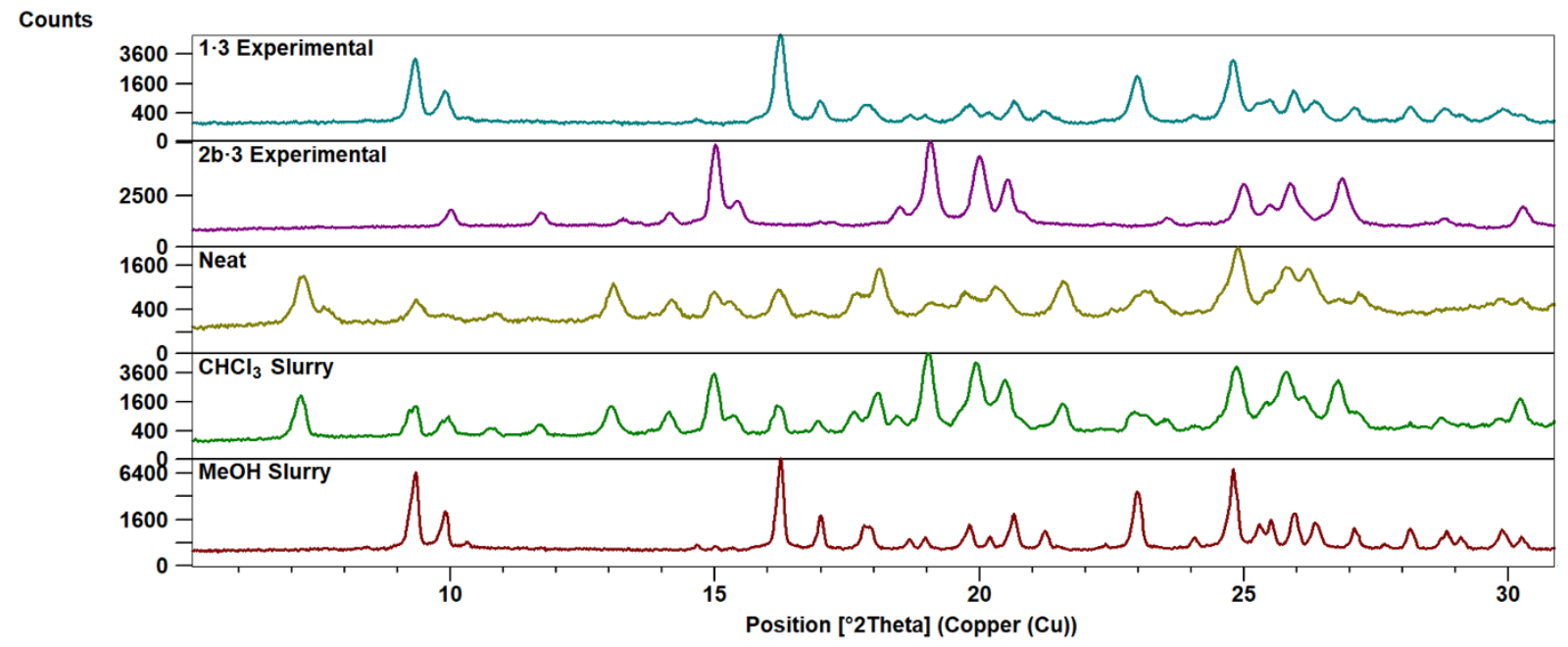

Figure S10. Comparison of neat grinding and slurry results for System B. When neat grinding is carried out, as well as after slurry experiments in chloroform, the ternary co-crystal is the major product (see also Figure S11), with small amounts of the two known co-crystals forming, similar to the LAG results. When slurry experiments are carried out in methanol, only the halogen-bonded co-crystal $\mathbf{1 \cdot 3}$ is formed. 


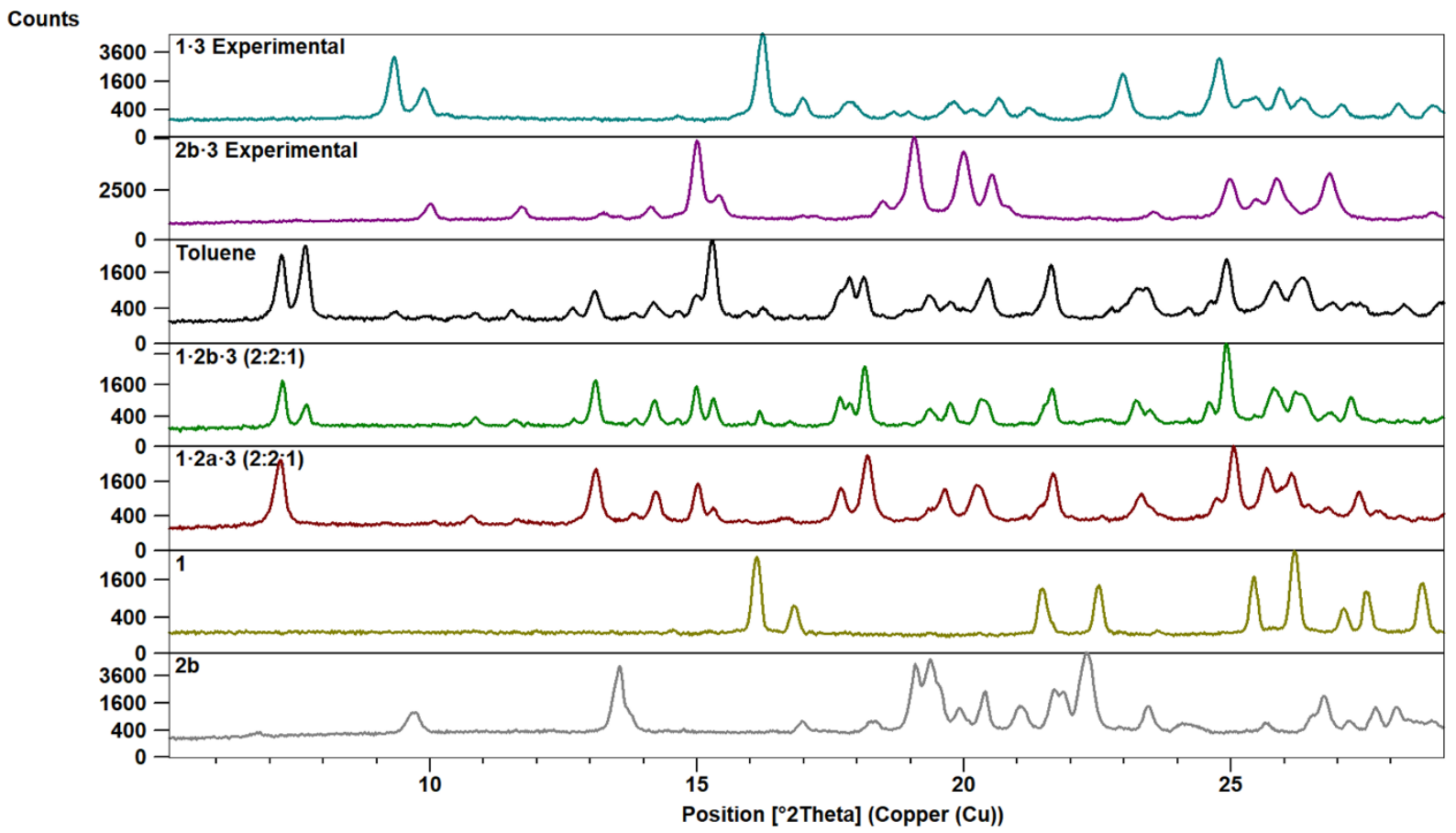

Figure S11. A closer look at one of the patterns containing the ternary co-crystal $\mathbf{1} \cdot \mathbf{2} \mathbf{b} \cdot \mathbf{3}$ shows that there are many peaks that cannot be assigned to either $\mathbf{1 \cdot 3}$ or $\mathbf{2 b} \cdot \mathbf{3}$ - the pattern obtained from grinding $\mathbf{1}, \mathbf{2 b}$, and $\mathbf{3}$ with toluene is used here as an example, shown in black (also contains $\mathbf{1 \cdot 3}$ and $\mathbf{2 b \cdot 3}$ ). We suspect that the ternary co-crystal contains $\mathbf{1}, \mathbf{2 b}$, and $\mathbf{3}$ in a 2:2:1 ratio. When the three starting materials are milled in this ratio, the pattern shown in red is obtained, which shows good correlation with the trace obtained from LAG. Furthermore, the pattern for this ternary co-crystal (red) matches the trace for the $\mathbf{1} \cdot \mathbf{2 a} \cdot \mathbf{3}$ ternary co-crystal, indicating that these are likely isostructural materials. 


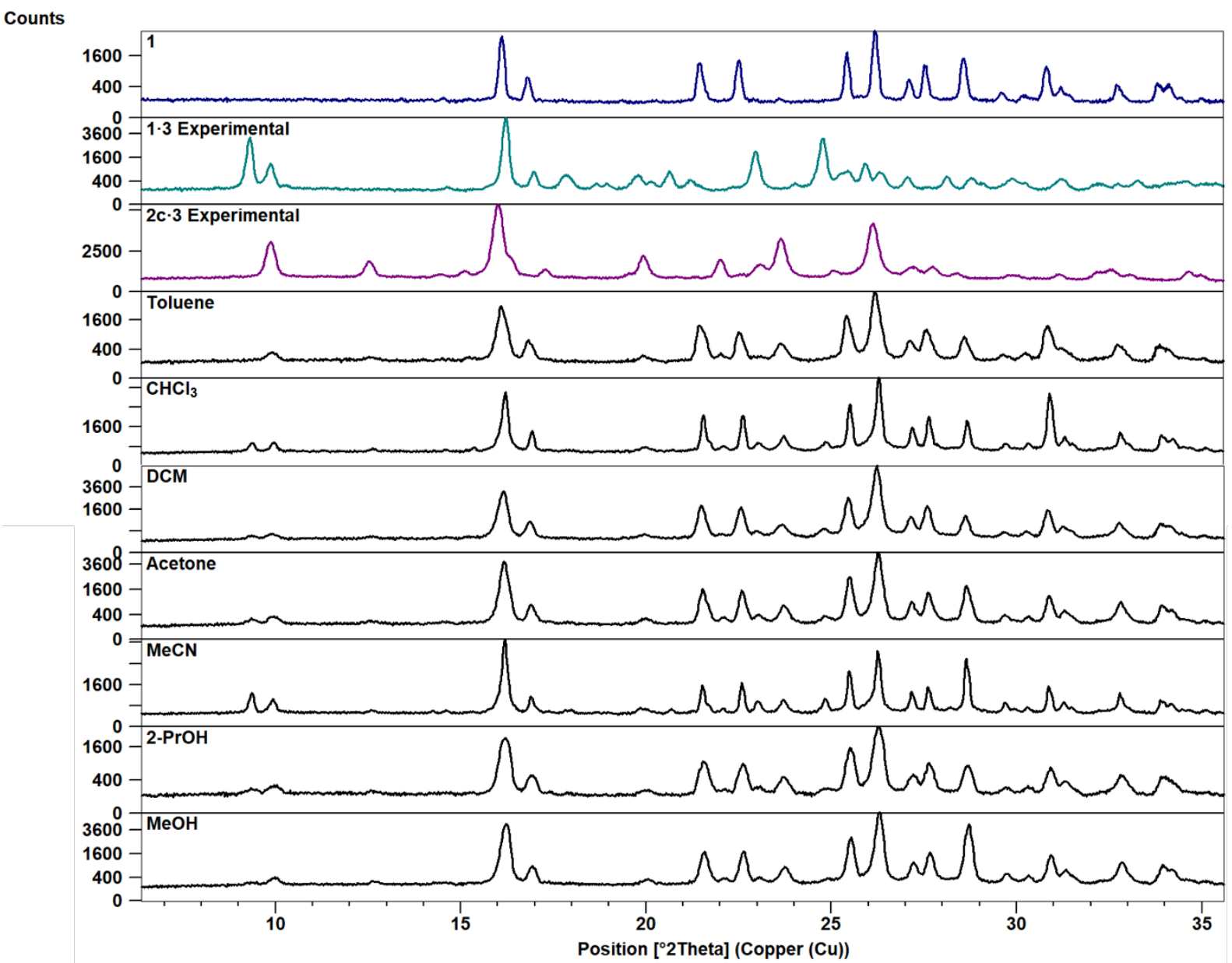

Figure S12. Comparison of LAG results, using seven different solvents, for System C. For each solvent used, small amounts of both the halogen bonded co-crystal $(\mathbf{1 \cdot 3})$ and the hydrogen-bonded co-crystal $(\mathbf{2 c} \cdot \mathbf{3})$ is formed, and a large amount of 1,4-diiodotetrafluorobenzene remains. 


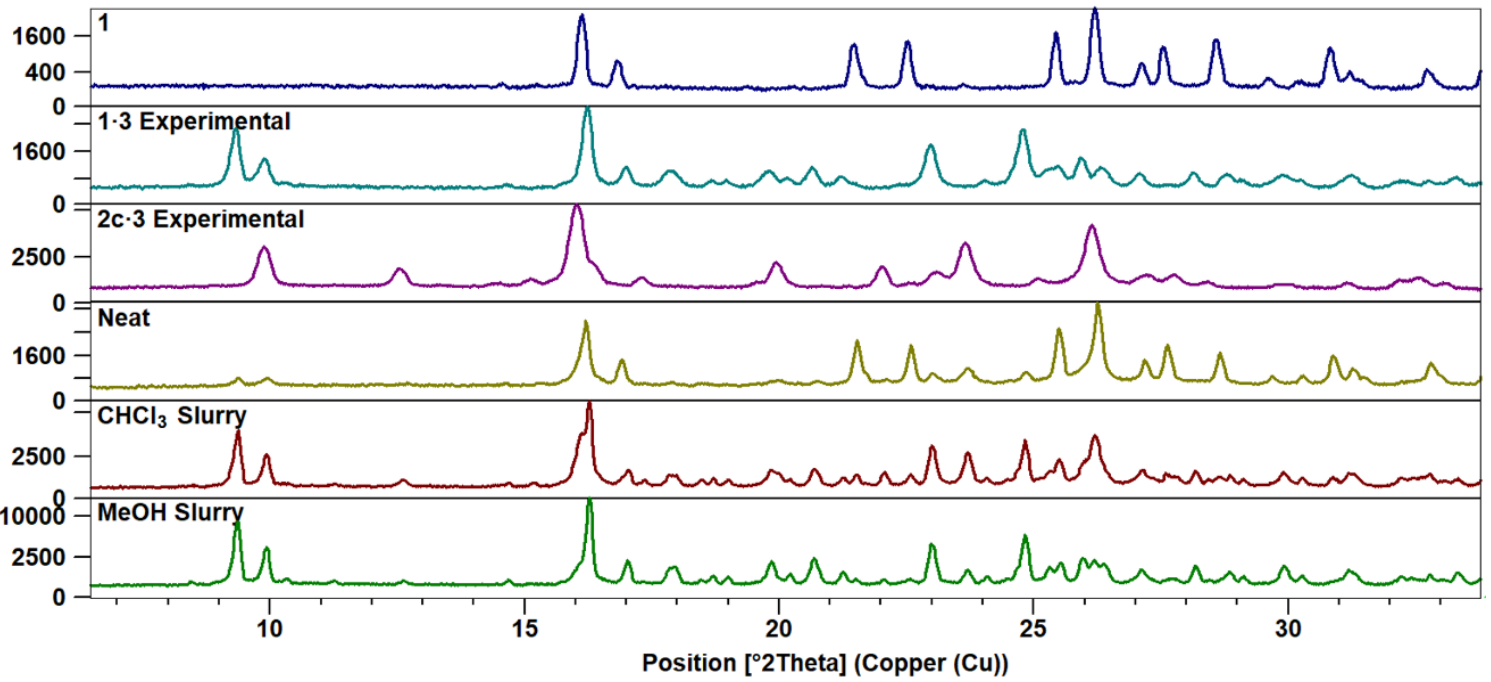

Figure S13. Comparison of neat grinding and slurry results for System C. When neat grinding is carried out, both $\mathbf{1} \cdot \mathbf{3}$ and $\mathbf{2 c} \cdot \mathbf{3}$ is formed, along with $\mathbf{1}$ being left over, similar to the LAG results. When slurry experiments are carried out in methanol and chloroform, similar results are obtained, but the halogenbonded co-crystal, $\mathbf{1} \cdot \mathbf{3}$, becomes more prominent, and much less unreacted starting material remains. The peaks corresponding to $\mathbf{2} \mathbf{c} \cdot \mathbf{3}$ similarly become more visible, e.g. around $12.6^{\circ}$.

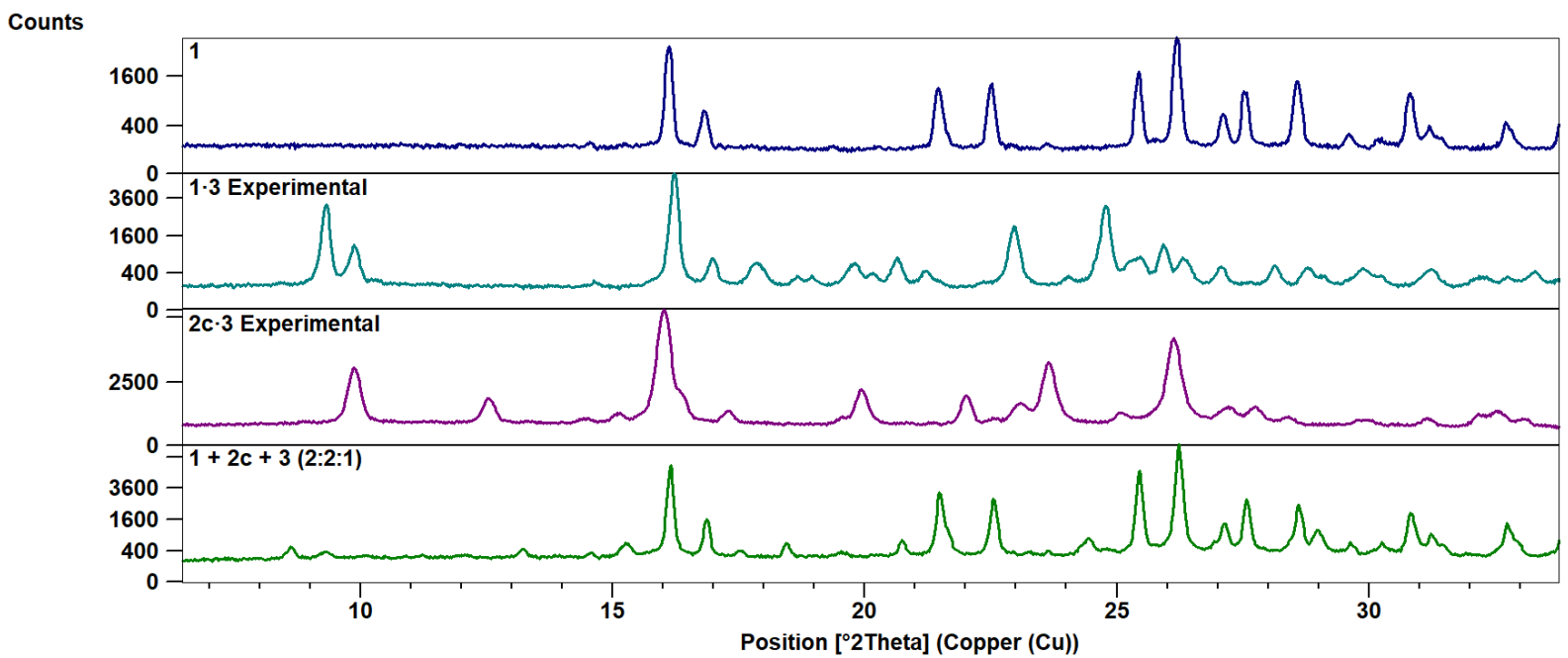

Figure S14. When a 2:2:1 ratio of $\mathbf{1}, \mathbf{2 c}$ and $\mathbf{3}$ are milled together, similar to what was done for Systems $\mathrm{A}$ and $\mathrm{B}$, the trace is dominated by left-over starting material $\mathbf{1}$. However, some new peaks do appear (e.g. peak at $8.6^{\circ}$ ), indicating another crystalline product that potentially exists. 


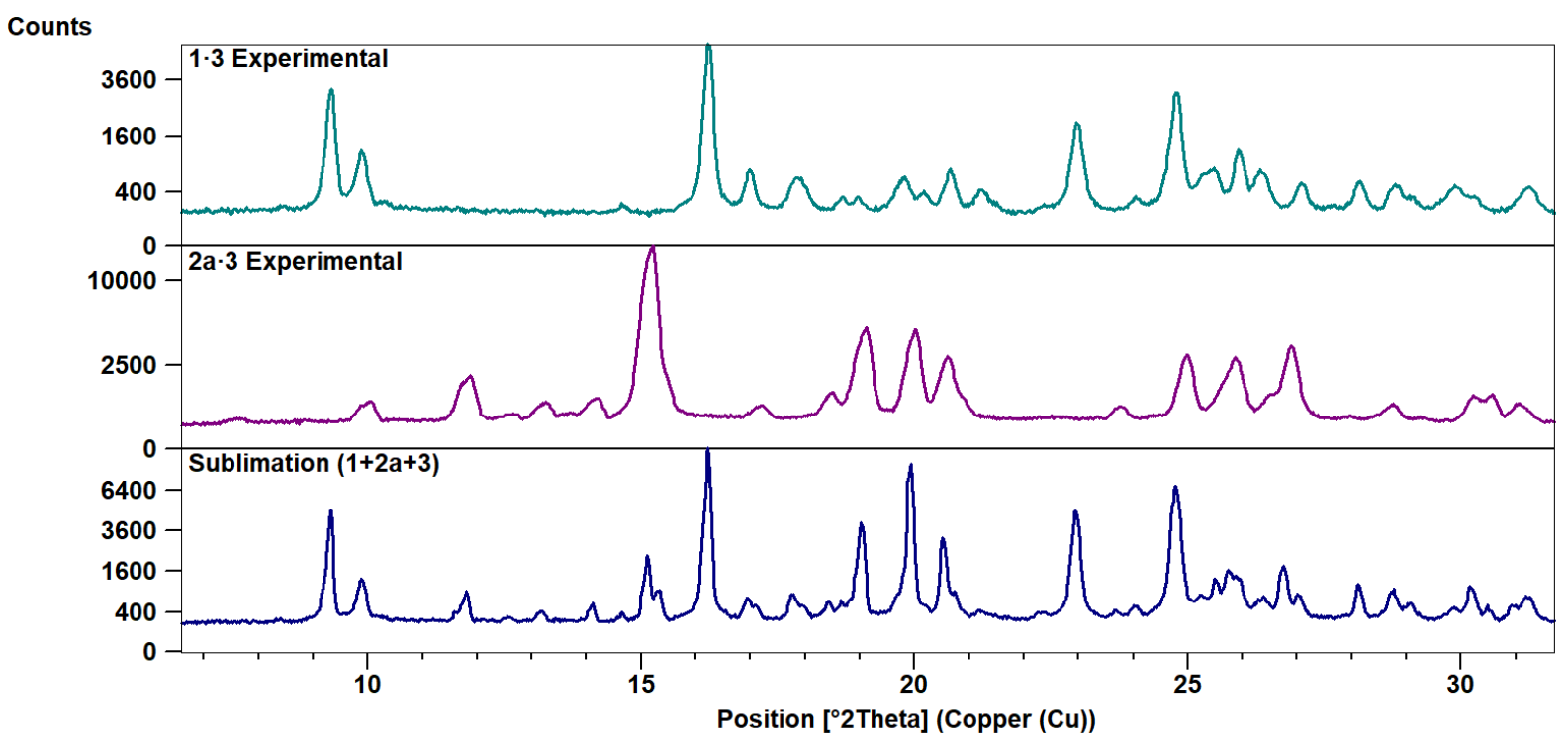

Figure S15. Results of selectivity tests by sublimation for System A. When a mixture of 1, 2a, and $\mathbf{3}$ is sublimed at $130{ }^{\circ} \mathrm{C}$, a mixture of $\mathbf{1 \cdot 3}$ and $\mathbf{2 a} \cdot \mathbf{3}$ is formed.

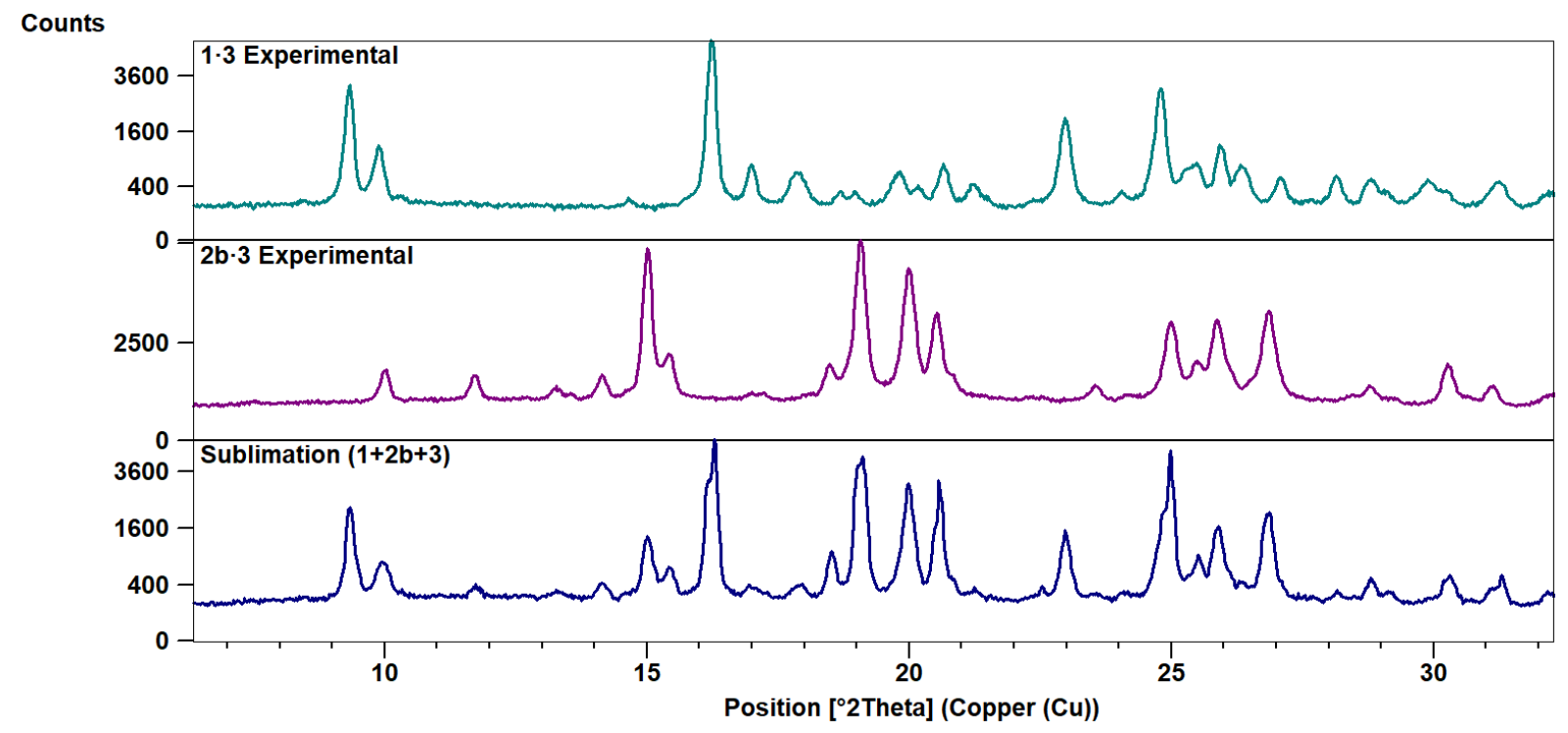

Figure S16. Results of selectivity tests by sublimation for System B. When a mixture of $\mathbf{1}, \mathbf{2 b}$, and $\mathbf{3}$ is sublimed at $130{ }^{\circ} \mathrm{C}$, a mixture of $\mathbf{1} \cdot \mathbf{3}$ and $\mathbf{2 b} \cdot \mathbf{3}$ is formed. 


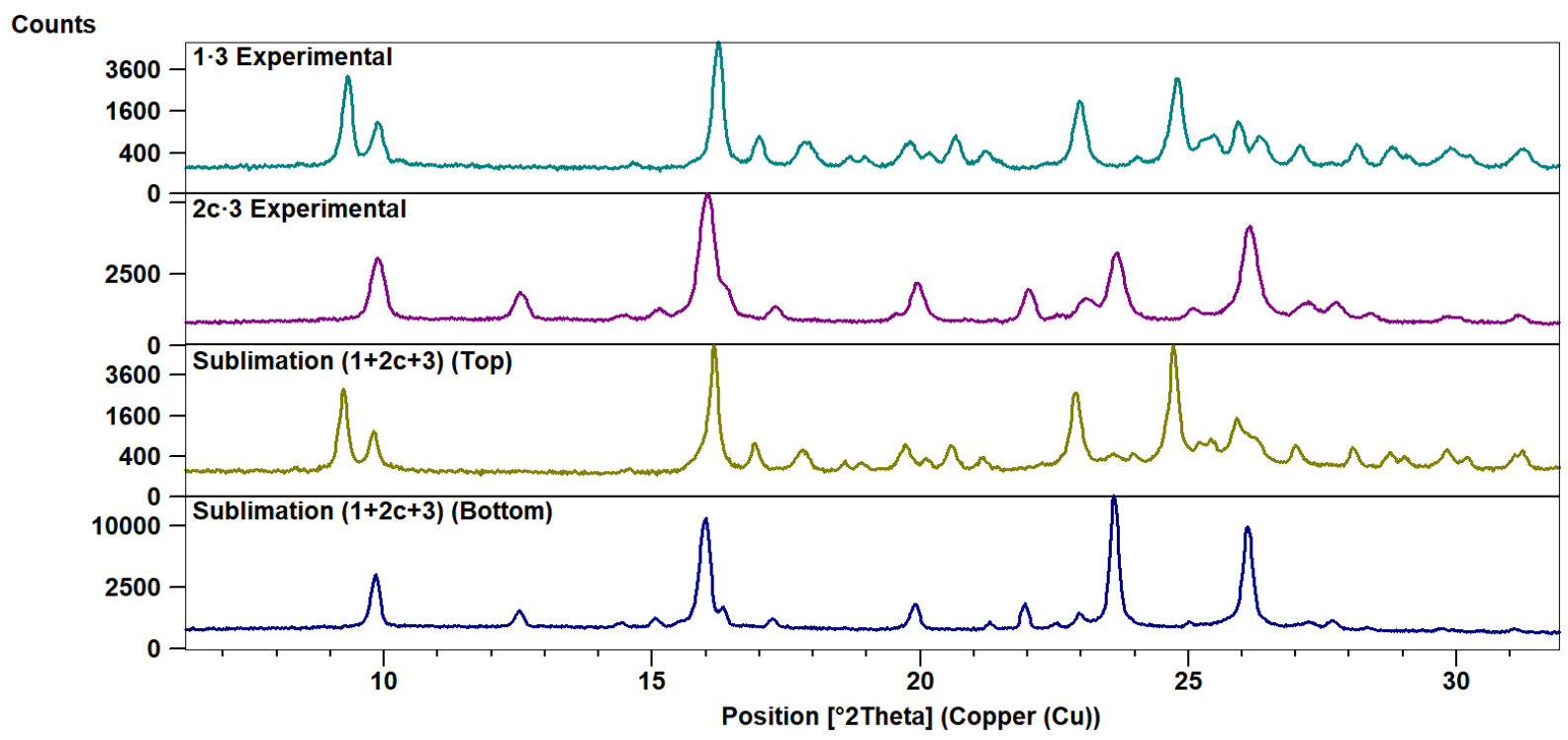

Figure S17. Results of selectivity tests by sublimation for System C. When a mixture of $\mathbf{1}, \mathbf{2 c}$, and $\mathbf{3}$ is sublimed at $130{ }^{\circ} \mathrm{C}$, separate bands of $\mathbf{1 \cdot 3}$ and $\mathbf{2 c} \cdot \mathbf{3}$ is formed. $\mathbf{1 \cdot 3}$ crystallizes at the top, and $\mathbf{2 c} \cdot \mathbf{3}$ forms below that.

\section{NMR}

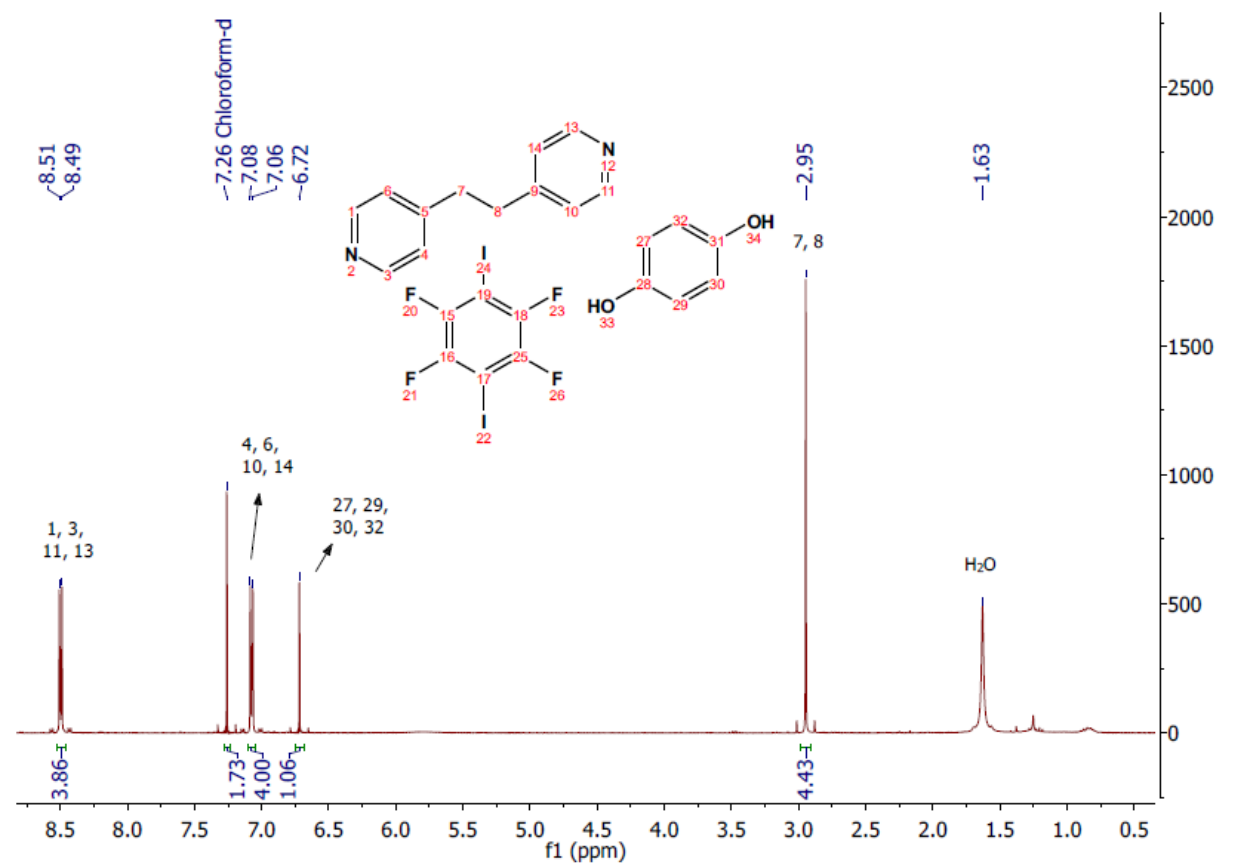

Figure S18. ${ }^{1} \mathrm{H}$ NMR spectrum (300 MHz) for the product obtained when a 2:2:1 mixture of 1, 2a and 3 was milled for 20 minutes, indicating that each individual molecule is still present in the product. Therefore, it can be concluded that no reaction occurred between the components. Peaks are referenced to the $\mathrm{CDCl}_{3}$ peak at $7.26 \mathrm{ppm}$. 


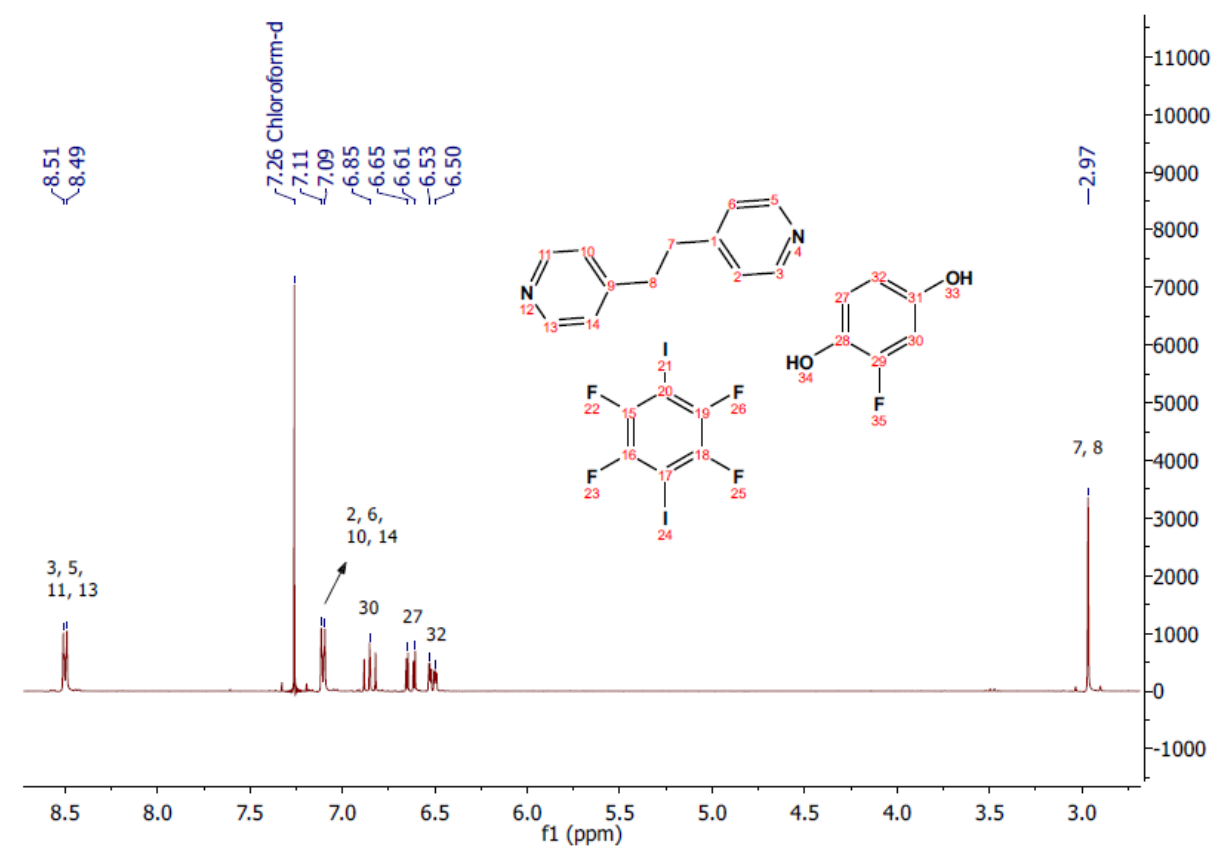

Figure S19. ${ }^{1} \mathrm{H}$ NMR spectrum $(300 \mathrm{MHz})$ for the product obtained when a 2:2:1 mixture of $\mathbf{1}, \mathbf{2 b}$ and 3 was milled for 20 minutes, indicating that each individual molecule is still present in the product. Therefore, it can be concluded that no reaction occurred between the components. Peaks are referenced to the $\mathrm{CDCl}_{3}$ peak at $7.26 \mathrm{ppm}$. 


\section{Thermal analysis}

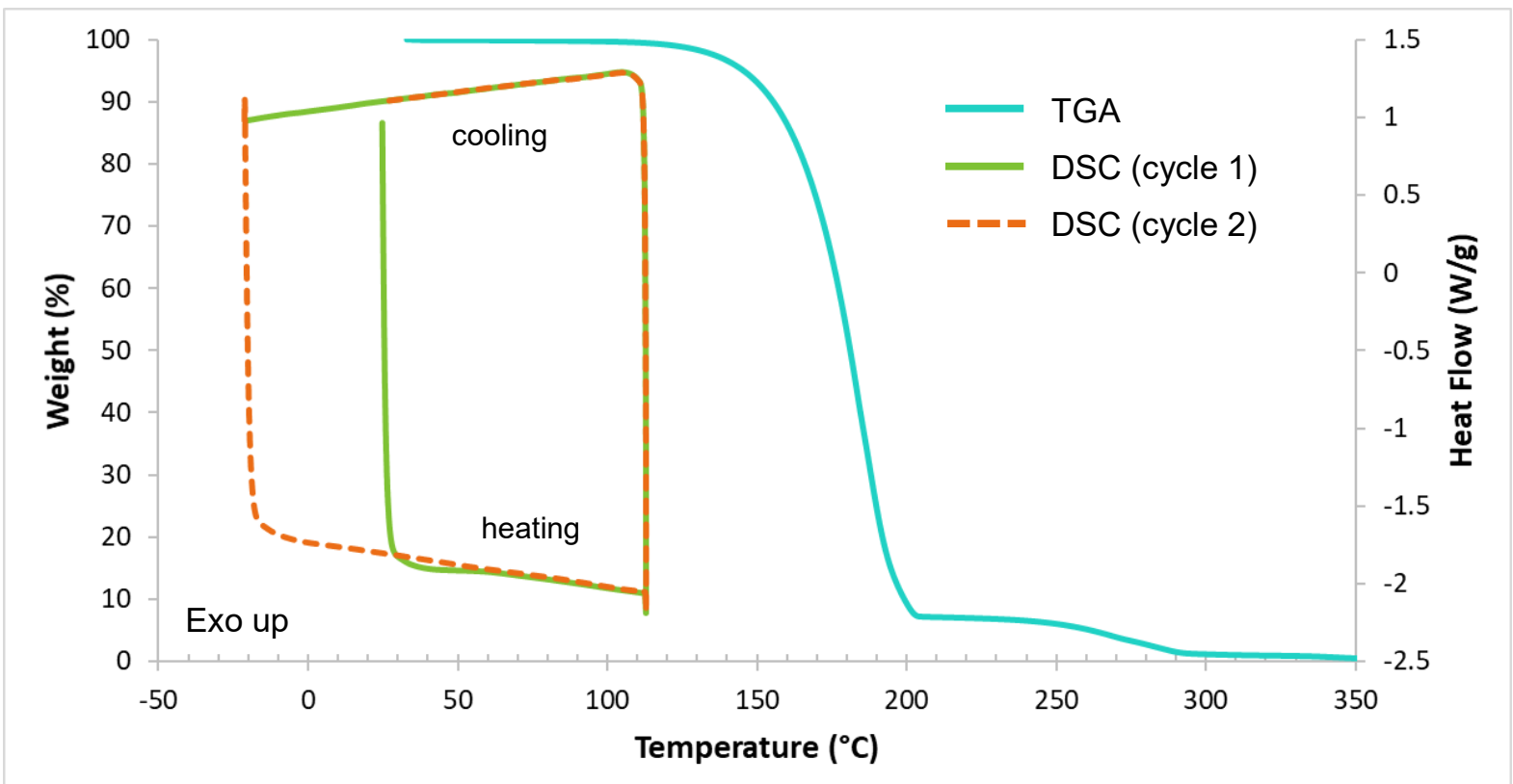

Figure S20. Thermal analysis results for 1·3. TGA trace shown in blue and DSC traces in green (cycle 1) and orange dashes (cycle 2).

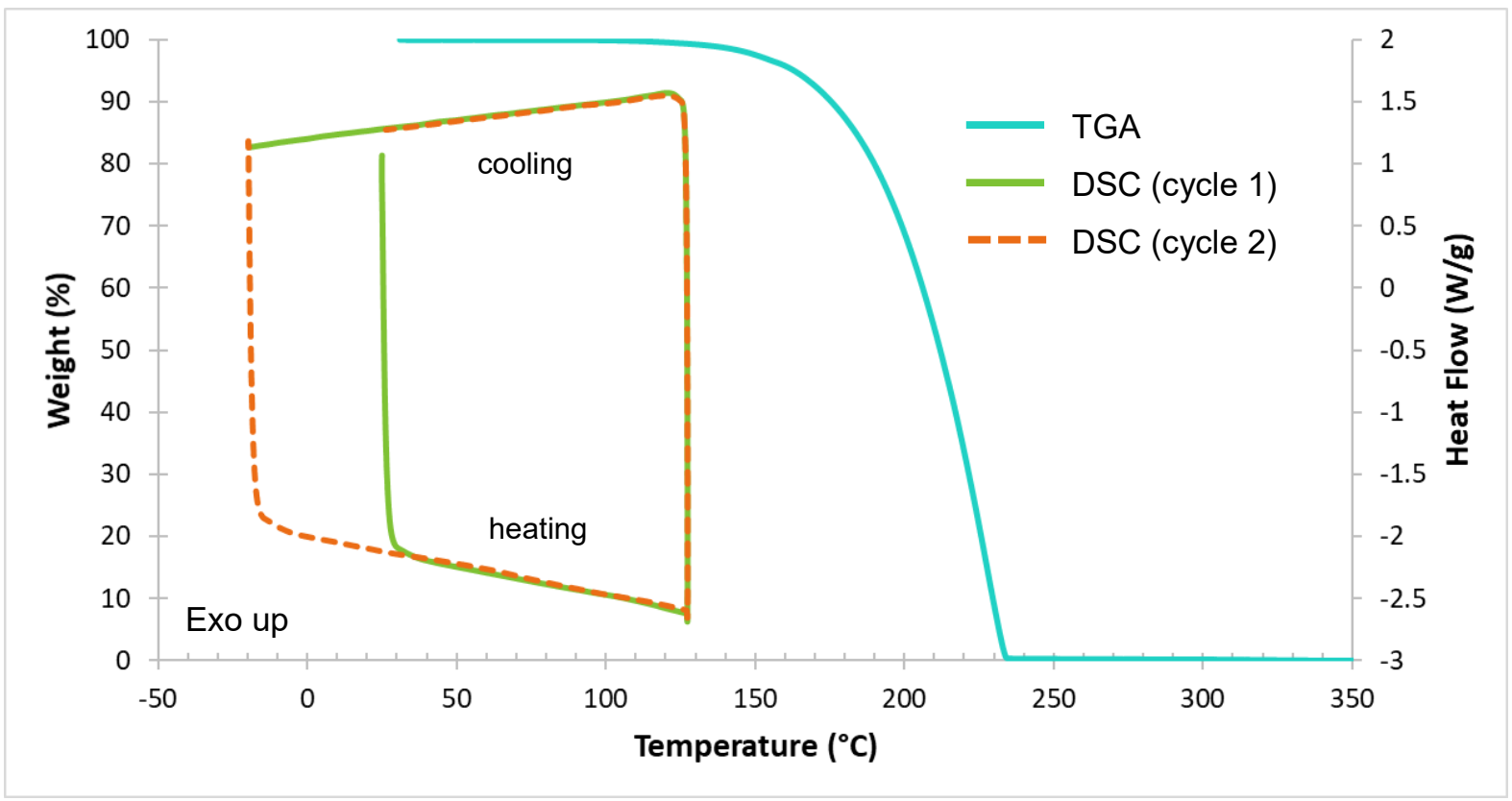

Figure S21. Thermal analysis results for $\mathbf{2 a \cdot 3}$. TGA trace shown in blue and DSC traces in green (cycle 1) and orange dashes (cycle 2). 


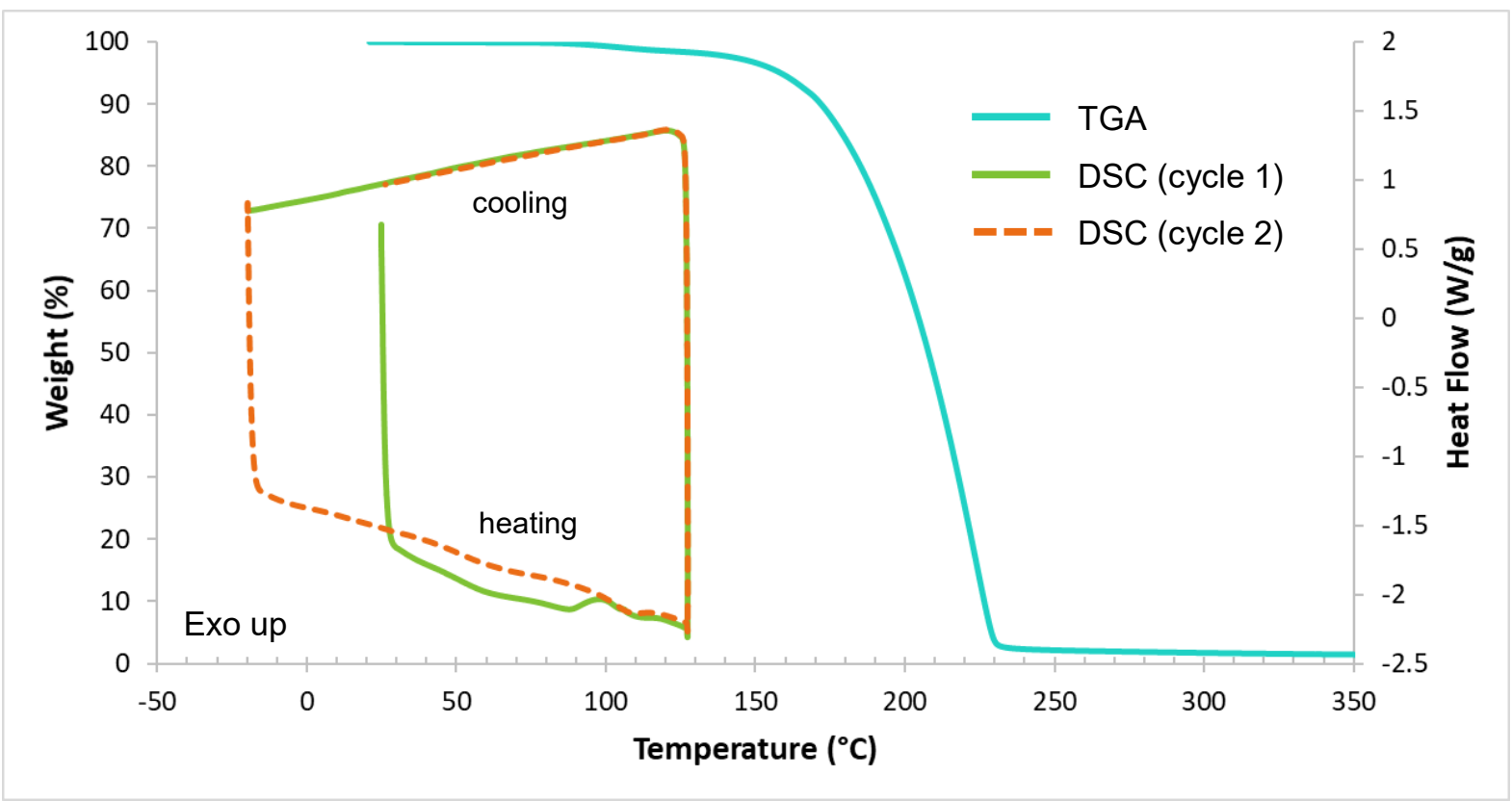

Figure S22. Thermal analysis results for $\mathbf{2 b} \cdot \mathbf{3}$. TGA trace shown in blue and DSC traces in green (cycle 1) and orange dashes (cycle 2).

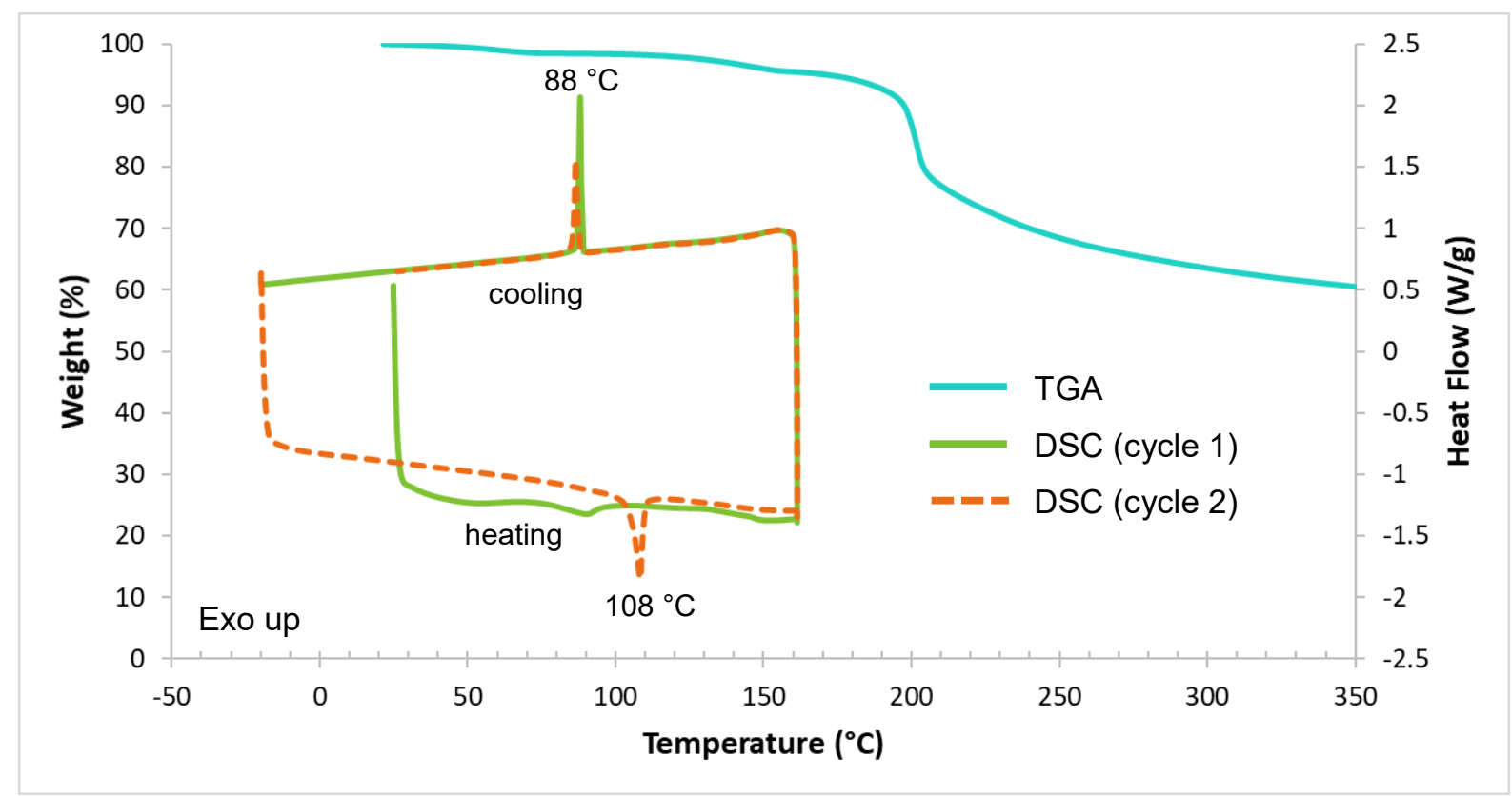

Figure S23. Thermal analysis results for $\mathbf{2 c \cdot 3}$. TGA trace shown in blue and DSC traces in green (cycle 1) and orange dashes (cycle 2). A melting point and subsequent crystallization upon cooling can be seen.

1. Christian Reichardt, Solvents and Solvent Effects in Organic Chemistry, Wiley-VCH Publishers, 3rd ed., 2003. DOI:10.1002/3527601791. 